\title{
PERCEPÇÃO SOBRE O ISOLAMENTO SOCIAL NO CONTEXTO DA PANDEMIA DE COVID-19 NO ESTADO DE PERNAMBUCO, BRASIL
}

\section{PERCEPTION ON SOCIAL ISOLATION IN THE CONTEXT OF THE PANDEMIC OF COVID-19 IN THE STATE OF PERNAMBUCO, BRAZIL}

\author{
Anselmo César Vasconcelos Bezerra \\ Instituto Federal de Educação, Ciência e Tecnologia de Pernambuco (IFPE) \\ anselmo@recife.ifpe.edu.br \\ Carlos Eduardo Menezes da Silva \\ Instituto Federal de Educação, Ciência e Tecnologia de Pernambuco (IFPE) \\ carlosmenezes@recife.ifpe.edu.br \\ Fernando Ramalho Gameleira Soares \\ Instituto Brasileiro de Geografia e Estatística (IBGE) \\ fernando.soares@ibge.gov.br
}

\begin{abstract}
RESUMO
O objetivo deste artigo é apresentar os resultados de uma pesquisa de opinião realizada com residentes do estado de Pernambuco sobre os fatores relacionados ao isolamento social durante a pandemia de COVID-19. Utilizou-se de um questionário construído no Google Forms e enviado pelas redes sociais: whatsapp, instagram e facebook. As perguntas buscavam evidenciar: perfil socioeconômico; situação de isolamento social; impactos do isolamento; expectativa do tempo que permanecerá em isolamento; e condições de habitação e saúde. As 7.593 respostas evidenciaram que $32 \%$ das pessoas estão em isolamento total; $57 \%$ em isolamento parcial; e $11 \%$ não estão isoladas. $88 \%$ acreditam na estratégia do isolamento. O convívio social foi o principal impacto percebido para $37 \%$ dos respondentes. $74 \%$ relataram algum nível de estresse; $21 \%$ pararam de ganhar dinheiro; $56 \%$ apresentaram alterações no sono e apenas $37 \%$ está fazendo algum exercício. Para $79 \%$ a residência é boa ou ótima para ficar isolado. Além disso, as condições de habitação demonstraram forte correlação com a possibilidade de ficar mais tempo em auto isolamento, o que no contexto do estado com mais de 800 mil pessoas vivendo em assentamentos subnormais ressalta a importância de estratégias direcionadas a este fator para ampliar a efetividade da estratégia de enfrentamento da pandemia.
\end{abstract}

Palavras-chaves: Distanciamento social. Novo Coronavírus. Saúde Mental.

\begin{abstract}
The purpose of this paper is to present the results of an opinion poll conducted with residents in the state of Pernambuco about factors related to social isolation during a COVID-19 pandemic. Use a questionnaire created on Google Forms and sent by social networks. The questionnaire aims to show: socioeconomic profile; situation of social isolation; isolation impacts; expectation of time separating in isolation; and housing and health conditions. The 7,593 responses showed that $32 \%$ are in total isolation; $57 \%$ in partial isolation; and $11 \%$ are not isolated. $88 \%$ believe in the isolation strategy. Social interaction was the main impact perceived by $37 \%$ of respondents. $74 \%$ reported some level of stress; $21 \%$ stopped making money; $56 \%$ reported changes in sleep; only $37 \%$ are doing any exercise. In addition, $79 \%$ consider their residence to be good or excellent. Furthermore housing conditions showed a strong correlation with the possibility of staying longer in selfisolation. In the context of the state with more than 800 thousand people living in subnormal settlements, the importance of strategies aimed at this factor is emphasized to increase the effectiveness of the pandemic coping strategy
\end{abstract}

Keywords: Social Distancing. New Coronovirus. Mental Health.

Recebido em: 02/05/2020

Aceito para publicação em: 15/05/2020. 


\section{INTRODUÇÃO}

No final do ano de 2019 uma nova doença surgiu na China causando sérios problemas respiratórios nos habitantes da Província de Wuhan. Essa doença, semelhante a uma pneumonia foi denominada de Sars-Covid-19 (WHO, 2020). No Brasil, o primeiro caso foi reportado na cidade de São Paulo no dia 25 de fevereiro de 2020 (CRODA e GARCIA, 2020; MORALES et al., 2020). Já no estado de Pernambuco, os primeiros casos foram registrados em 05 de março de 2020 , um casal que havia retornado da Itália (PERNAMBUCO, 2020). Após esses registros, o governo do estado e algumas prefeituras começaram a publicar decretos com medidas para o enfrentamento da COVID-19, entre as estratégias: a suspensão de aulas em escolas públicas e particulares, cancelamento de eventos e orientações à população sobre distanciamento e isolamento social (PERNAMBUCO, 2020a).

Após um mês do registro dos primeiros casos no estado, a maior parte da população já havia sido impactada de alguma forma pelas medidas de distanciamento e isolamento social, especialmente na Região Metropolitana do Recife, onde estavam concentrados cerca de $80 \%$ dos casos confirmados e óbitos (PERNAMBUCO, 2020b). Dados revelaram que o índice de isolamento social no estado variou entre $50 \%$ e $53 \%$ entre 16 de março e 15 de abril, com tendência a um maior isolamento após a publicação de decretos restritivos à circulação de pessoas por parte das autoridades (ENDO et al., 2020). Todavia, as medidas adotadas pelo governo começaram a ser questionadas, uma vez que parte da população não estava convencida do risco da doença, bem como estava sendo impactada na perda do poder de compra e consequentemente na própria subsistência.

Assim, o isolamento social passou a ser um dos assuntos mais comentados pela mídia e nos grupos virtuais de interação social. A partir dos dados de localização de usuários de celular foi possível verificar que no dia 12 de abril de 2020, Pernambuco tinha um índice de isolamento social de 50,6\%, ocupando a segunda posição nacional, atrás apenas do Distrito Federal com 56\% (DIÁRIO DE PERNAMBUCO, 2020). Mesmo assim, o estado figura entre os principais em número de casos confirmados $\left(4^{\circ}\right)$ e com uma alta taxa de letalidade da doença $(8,4 \%)$, acima da média nacional de $6,8 \%$ (BRASIL, 2020).

Essa aparente incoerência entre um maior isolamento social e um alto número de casos, não necessariamente indica que a estratégia de isolamento seja ineficaz, uma vez que existe um alto nível de subnotificação de casos, além de diferentes percentuais de testagem da população nos estados brasileiros (BRASIL, 2020). Além disso, projeções indicam que se não fosse o isolamento social, os números de casos e óbitos poderiam ser muito maiores (ANDERSON et al., 2020).

Ainda assim diversos são os fatores que influenciam na decisão de adotar ou não o auto isolamento. No estado do Ceará observou-se através de pesquisa de percepção que as crenças da população variaram em relação a sexo, idade e escolaridade da população. Por exemplo, as mulheres confiam mais nos resultados do isolamento, os mais jovens estão menos isolados e os menos escolarizados acreditam que têm menos riscos de contrair o vírus em relação aos mais escolarizados (LIMA et al., 2020).

Em outros países, verificou-se que a população está disposta a permanecer em isolamento social o tempo que for necessário, mas admitem certa frustração quando os governos sinalizam para a flexibilização do isolamento e depois recuam da decisão (BRISCESE, 2020). Em outro caso, observou-se que a população mais pobre até desejaria está isolada nesse momento, entretanto esse grupo tem sua chance diminuída em comparação aos grupos com maior renda (ATCHIMSON, 2020). Outros estudos já mostram que há uma correlação entre os impactos do processo de isolamento social e o aumento de estresse, ansiedade e depressão das pessoas (QUIO et al., 2020; XIAO et al., 2020, ORNELL et al., 2020). Também, que pessoas em isolamento social confiam fortemente nessa estratégia (DATAFOLHA, 2020), mas colocam em xeque as decisões fracas dos governos em relação às medidas mais corretas para combater a Pandemia (FETZER et al., 2020). Também já existem estudos que associam às condições de habitação durante o isolamento com a propensão maior ou menor em aderir à medida (PANCANI et al., 2020).

Diante desse cenário, essa pesquisa objetivou compreender a percepção da população pernambucana em relação aos fatores que influenciam na decisão pelo auto isolamento durante a pandemia de COVID-19, e os possíveis impactos oriundos desse processo. Neste estudo, o termo isolamento social foi usado no mesmo sentido de distanciamento social, pois observou-se que a população e a mídia estavam fazendo mais referência ao isolamento. 


\section{MÉTODO}

A pesquisa foi realizada através de um questionário com dezessete perguntas objetivas no formato de pesquisa de opinião, conforme normas da resolução 510/16 (BRASIL, 2016). Os participantes não precisavam se identificar. A pesquisa captou respostas da população do estado de Pernambuco que dispõe de algum equipamento digital com acesso à internet, configurando uma amostra não probabilística com viés de conveniência.

O formulário de coleta foi construído na plataforma Formulários Google ${ }$ e divulgado via internet, através das redes sociais entre os dias 6 e 8 de abril de 2020. Ao todo, obtiveram-se 7.593 respostas. Só foram considerados válidos os questionários obtidos de respondentes com dezoito anos ou mais. O questionário foi estruturado de forma que conseguisse captar as dimensões que ajudassem na compreensão da percepção social em relação ao isolamento no contexto da Pandemia.

No primeiro bloco de questões abordou-se aspectos referente ao perfil socioeconômico das pessoas, com as variáveis de sexo, idade, nível de escolaridade e faixa de renda. Na segunda parte questionou-se como o isolamento está impactando a vida das pessoas a partir das seguintes variáveis: se está ou não isolado, por que não está isolado, qual o principal impacto do isolamento, como o isolamento está afetando a renda/gastos e a saúde. No terceiro bloco focou-se nas condições de habitabilidade no isolamento: número de pessoas na residência, percepção de conforto da residência, presença ou ausência de área aberta e/ou verde na residência. Por fim, questionou-se sobre a expectativa das pessoas em relação ao tempo máximo que acreditam suportar na condição de isolamento social durante a pandemia.

A análise foi feita a partir da tabulação dos dados na planilha Excel e do software STATA® 13. Para tanto, foram analisadas as correlações entre as variáveis do questionário e realizado o teste Quiquadrado de Pearson, para as variáveis categóricas. A aplicação do teste objetivou verificar a associação entre variáveis categóricas, considerando para tanto um nível de confiança desejável de 99\% para análise do valor crítico da distribuição qui-quadrado, e um p-valor maior que 0,01 (nível de significância de 1\%), nos diferentes graus de liberdade.

Após realizados os testes de associação, definiu-se que para a discussão deste artigo, serão apresentados os achados de maior significância relacionados aos fatores que parecem influenciar na decisão pelo auto isolamento e de maior permanência na residência durante à Pandemia.

Destaca-se que a pesquisa não teve o objetivo de extrapolar seus resultados para a população do estado como um todo, uma vez que a amostra teve um viés de conveniência e a forma de responder estava atrelada a necessidade do uso de internet pelos participantes, o que gerou uma concentração de respostas em determinados grupos populacionais, corroborando com os dados da pesquisa do Instituto Brasileiro de Geografia e Estatística (IBGE) que demonstrou que 23\% da população nordestina ainda não tem acesso à internet (BRASIL, 2020a). Tal fato, apesar de ser uma limitação do estudo, não inviabiliza sua divulgação, visto que os achados representam em certa medida a percepção de uma parcela significativa de respondentes no estado de Pernambuco, e o retrato apresentado pode ajudar na compreensão dos impactos do isolamento pela ótica das pessoas que estão sendo afetadas de formas diferentes.

\section{RESULTADOS E DISCUSSÕES}

Do total de 7.953 respostas da amostra, $70 \%$ das pessoas foram do sexo feminino, enquanto $30 \%$ do sexo masculino. Em relação às faixas de renda, observou-se que $37 \%$ das pessoas estava na faixa de até 2 salários mínimos, $32 \%$ na faixa que recebe entre 2 e 5 salários mínimos, $15 \%$ recebem entre 5 e 8 salários mínimos e 16\% ganham acima de 8 salários mínimos.

Sobre escolaridade, os dados revelaram um maior quantitativo de participantes com nível superior (35\%) e pós-graduação (49\%), enquanto $15 \%$ dos respondentes possuem ensino médio e $1 \%$ ensino fundamental. Esse dado pode ser explicado pelo formato da pesquisa, divulgação via redes sociais e internet, relacionados com o percentual da população que não dispõe desse recurso.

No universo amostrado, $32 \%$ afirmaram que estão em isolamento total, ou seja, não saem de suas residências; $57 \%$ das pessoas estão em isolamento parcial, termo que significa, nesta pesquisa, sair de casa apenas para comprar alimentos e medicamentos; e $11 \%$ não estão isoladas, total ou parcialmente. Observou-se em pesquisas utilizaram dados de localização de celulares, que a média 
de isolamento social no estado foi de $51,5 \%$ no período de 16 de março a 15 de abril (ENDO et al., 2020).

De forma geral, $88 \%$ dos pernambucanos acreditam que o isolamento reduz o número de vítimas da COVID-19, enquanto $8 \%$ não tem certeza, e apenas $4 \%$ responderam que o isolamento não é capaz de reduzir o número de vítimas da COVID-19. Dados nacionais do início do mês de abril mostraram que $76 \%$ das pessoas eram favoráveis ao isolamento (DATAFOLHA, 2020), entretanto numa pesquisa mais recente, observou-se que $56 \%$ dos brasileiros não acreditam que o isolamento será capaz de conter a propagação da COVID-19 (IPSOS, 2020). As divergências entre os dados mostra a complexidade do cenário e as diferentes percepções das pessoas frente à Pandemia.

Para $37 \%$ dos respondentes, o convívio social é o principal aspecto que está sendo afetado pelo isolamento, já $27 \%$ das pessoas relataram o aspecto financeiro como o mais impactante, $17 \%$ dos respondentes informam que o isolamento não está ocasionando nenhum tipo de impacto e $10 \%$ apontam outros fatores que estão sendo impactados, apenas $8 \%$ atribuem o principal impacto à própria saúde. Possivelmente, a representação do principal impacto relatado está relacionado ao convívio social tem a ver com o perfil majoritário dos respondentes, contudo pesquisas revelam que a ruptura das relações sociais em função da COVID-19 é um fator sempre muito citado por pessoas em isolamento (FETZER et al., 2020). Os impactos financeiros estão mais presentes, entre os grupos que perderam o emprego ou comerciantes e autônomos.

Quando questionou-se sobre o impacto do isolamento social na renda/gastos das pessoas, as respostas foram as seguintes: para $31 \%$ das pessoas, o isolamento não está impactando a renda/gastos, já 33\% dos respondentes afirmam que estão economizando dinheiro, $15 \%$ das pessoas estão gastando mais dinheiro nesse período, enquanto $21 \%$ afirmaram que pararam de ganhar dinheiro em função do isolamento social. Pesquisa do Instituto Locomotiva no início do mês de abril demonstrou que $51 \%$ dos brasileiros declararam alguma perda de renda (INSTITUTO LOCOMOTIVA, 2020). Em função dessa perda no poder de consumo das famílias, o governo federal criou um auxílio emergencial no valor de 600 reais mensais, entretanto a forma de pagamento desse auxílio tem gerado muitas filas e aglomerações em frente aos bancos e casas lotéricas, o que pode ser um catalisador na disseminação do vírus.

Inquiridos se o isolamento estava gerando algum estresse no ambiente doméstico, $26 \%$ disseram que não estavam sentindo nenhum estresse em função do isolamento, $56 \%$ relataram estar sentindo um pouco de estresse e 18\% afirmaram que o isolamento tem gerado muito estresse no ambiente doméstico. Os dados mostram que $74 \%$ estão estressadas com o contexto do isolamento social, o que já é esperado (ORNELL et al., 2020). Esse dado é importante, pois o que vai suceder a Pandemia pode estar relacionado com um aumento significativo nos casos de ansiedade, transtorno de pânico e depressão (BROOKS et al., 2020).

Investigou-se também como está o sono das pessoas diante desse momento de isolamento social. $41 \%$ dos respondentes afirmaram que estão mantendo o número de horas de sono que já dormiam antes do isolamento. Entretanto, $56 \%$ relataram alguma modificação nas horas de sono, sendo $28 \%$ dormindo mais horas que o habitual e 32\% dormindo menos que o habitual. Há uma relação estreita entre o aumento do estresse e a diminuição da qualidade do sono durante a pandemia (XIAO et al., 2020). Em relação à atividade física, $37 \%$ das pessoas está fazendo algum exercício e $63 \%$ não estão. No contexto da Pandemia, está acontecendo mudanças bruscas na vida das pessoas, mas a manutenção de hábitos, como alimentação saudável, atividade física e higiene do sono são fundamentais para mitigar os efeitos negativos na saúde (OLIVEIRA et al., 2020).

Quando questionou-se sobre a percepção das pessoas em relação às condições de habitabilidade, as respostas mostraram que $79 \%$ consideram sua residência boa ou ótima em relação aos fatores: tamanho, abastecimento de água e ventilação, enquanto apenas $21 \%$ consideram a residência como regular, ruim ou péssima. $61 \%$ também responderam que a residência possui alguma área aberta (terraço, quintal, área verde), contra $39 \%$ que não possuem. Dos que responderam que possuem alguma área aberta em casa, $64 \%$ acham que isso ajuda muito a conviver no período de isolamento. Pesquisa realizada com italianos demonstrou que condições físicas inadequadas das habitações durante o isolamento social aumentam os níveis de estresse e ansiedade dos moradores (PANCANI et al., 2020). 
Quando perguntados sobre o tempo máximo que aguentariam ficar isolados, os dados revelaram que $17 \%$ das pessoas afirmaram que não conseguem ficar um mês inteiro nesta condição, $19 \%$ responderam que conseguem ficar entre um e dois meses, $4 \%$ acreditam que conseguem ficar mais de dois meses, porém $60 \%$ está disposta a ficar o tempo que for necessário nesta condição para enfrentar à pandemia. Esse é um dado relativo, que tende a mudar com o tempo, à medida que as pessoas ficam mais dias isoladas sofrendo os impactos correlatos. Sobre isso, dados da população inglesa revelaram que os grupos de maior renda possuem mais tolerância a um maior tempo isolados do que os grupos de menor renda (ATCHIMSON et al., 2020).

Quando analisadas as correlações entre as variáveis é possível observar que a maior parte dos respondentes, $60 \%$ do total estavam no período avaliado dispostos a permanecer o tempo que fosse necessário em auto isolamento para conter a pandemia. Ainda assim, quando analisados em separado, de acordo com o principal impacto percebido, esse percentual cai para $47 \%$ entre aqueles cujo impacto financeiro é considerado o mais importante (Tabela 1).

Tabela 1 - Relação entre o tempo a mais que está disposto a estar em auto isolamento e a percepção do principal impacto

\begin{tabular}{|c|c|c|c|c|c|c|}
\hline \multirow[b]{2}{*}{$\begin{array}{l}\text { Tempo a mais disposto a } \\
\text { estar em auto isolamento }\end{array}$} & \multicolumn{6}{|c|}{ Principal Impacto do Isolamento } \\
\hline & Nenhum & $\begin{array}{l}\text { Convívio } \\
\text { social }\end{array}$ & Financeiro & Saúde & Outro & Total \\
\hline Menos de 1 Mês & $\begin{array}{r}63 \\
4.83 \%\end{array}$ & $\begin{array}{r}343 \\
12.03 \%\end{array}$ & $\begin{array}{r}561 \\
2754 \%\end{array}$ & $\begin{array}{r}126 \\
2019 \%\end{array}$ & $\begin{array}{r}107 \\
13.77 \%\end{array}$ & $\begin{array}{r}1.200 \\
15.80 \%\end{array}$ \\
\hline De 1 a 2 Meses & $\begin{array}{r}191 \\
14,65 \%\end{array}$ & $\begin{array}{r}638 \\
22,38 \%\end{array}$ & $\begin{array}{r}469 \\
23,02 \%\end{array}$ & $\begin{array}{r}122 \\
19,55 \%\end{array}$ & $\begin{array}{r}112 \\
14,41 \%\end{array}$ & $\begin{array}{r}1.532 \\
20,18 \%\end{array}$ \\
\hline Mais de 2 Meses & $\begin{array}{r}49 \\
3,76 \%\end{array}$ & $\begin{array}{r}104 \\
3,65 \%\end{array}$ & $\begin{array}{r}49 \\
2,41 \%\end{array}$ & $\begin{array}{r}21 \\
3,37 \%\end{array}$ & $\begin{array}{r}25 \\
3,22 \%\end{array}$ & $\begin{array}{r}248 \\
3,27 \%\end{array}$ \\
\hline Tempo necessário & $\begin{array}{r}1.001 \\
76,76 \%\end{array}$ & $\begin{array}{r}1.766 \\
61,94 \%\end{array}$ & $\begin{array}{r}958 \\
47,03 \%\end{array}$ & $\begin{array}{r}355 \\
56,89 \%\end{array}$ & $\begin{array}{r}533 \\
68,60 \%\end{array}$ & $\begin{array}{r}4.613 \\
60,75 \% \\
\end{array}$ \\
\hline Total & $\begin{array}{l}1.304 \\
100 \%\end{array}$ & $\begin{array}{l}2.851 \\
100 \%\end{array}$ & $\begin{array}{l}2.037 \\
100 \%\end{array}$ & $\begin{array}{r}624 \\
100 \%\end{array}$ & $\begin{array}{r}777 \\
100 \%\end{array}$ & $\begin{array}{l}7.593 \\
100 \%\end{array}$ \\
\hline
\end{tabular}

Teste Qui-quadradado: $X^{2}=495,01 ; p<0.001$

Fonte: Elaborado pelos autores com base em dados primários obtidos em formulário eletrônico.

Esses dados provavelmente estão relacionados ao fato de que estas pessoas não têm a possibilidade de manter a sua renda em trabalhos remotos, o que leva a perceber esse como principal impacto e ao mesmo tempo terem uma disposição de estar em auto isolamento por um período menor.

Ainda assim, durante o primeiro mês das medidas de isolamento, foi possível observar uma queda considerável na mobilidade das pessoas no estado, chegando a uma redução de mais de $60 \%$ em locais de compras e parques e um aumento de mais de $20 \%$ da presença em residências (GOOGLE, 2020). Essas variações encontraram seus pontos mais acentuados em datas imediatamente posteriores aos decretos estaduais de proibição da circulação e fechamento desses estabelecimentos comerciais não essenciais (ENDO et al, 2020). Porém, esses dados de mobilidade estão se alterando $\mathrm{e}$ até meados de abril a mobilidade de pessoas em estabelecimentos comerciais e locais de transporte público chegava a $50 \%$ (GOOGLE, 2020), abaixo da meta considerada ideal de $70 \%$ pelo estado.

Essa disposição em estar pelo tempo necessário em isolamento está relacionada com a percepção da imensa maioria dos respondentes $89,3 \%$ (6781 pessoas) de que a estratégia de isolamento social é sim eficaz e capaz de reduzir o número de vítimas na Pandemia de COVID-19 (Tabela 2). Esse é um dado extremamente interessante, uma vez que poderia ser um indicador de uma maior propensão 
a praticar o auto isolamento, caso outras condições não dificultassem a adesão a estratégia por parte de um contingente significativo de pessoas no estado.

Dentre os diferentes fatores que influenciam na capacidade dos indivíduos em adotar o auto isolamento, pode-se destacar a qualidade das residências. Dentre os respondentes do questionário, pode-se observar que o percentual de pessoas dispostas a ficar o tempo necessário em isolamento é crescente de acordo com a percepção da qualidade da residência. Da mesma forma, a disposição em ficar menos de 1 mês em isolamento é crescente quão pior é a percepção da qualidade da residência (Tabela 3).

Tabela 2- Relação entre o tempo a mais que está disposto a estar em auto isolamento e a percepção da eficácia do isolamento em reduzir o número de vítimas da pandemia de COVID-19

\begin{tabular}{|c|c|c|c|c|}
\hline \multirow{2}{*}{$\begin{array}{c}\text { Tempo a mais disposto a estar } \\
\text { em auto isolamento }\end{array}$} & \multicolumn{4}{|c|}{ Isolamento reduz vítimas } \\
\hline & Não & Ivez s s & & tal \\
\hline \multirow[t]{2}{*}{ Menos de 1 Mês } & 184 & 284 & 732 & 1.200 \\
\hline & $76,67 \%$ & $49,65 \%$ & $10,79 \%$ & $15,80 \%$ \\
\hline \multirow[t]{2}{*}{ De 1 a 2 Meses } & 16 & 102 & 1.414 & 1.532 \\
\hline & $6,67 \%$ & $17,83 \%$ & $20,85 \%$ & $20,18 \%$ \\
\hline \multirow[t]{2}{*}{ Mais de 2 Meses } & 3 & 13 & 232 & 248 \\
\hline & $1,25 \%$ & $2,27 \%$ & $3,42 \%$ & $3,27 \%$ \\
\hline \multirow[t]{2}{*}{ Tempo necessário } & 37 & 173 & 4.403 & 4.613 \\
\hline & $15,42 \%$ & $30,24 \%$ & $64,93 \%$ & $60,75 \%$ \\
\hline \multirow[t]{2}{*}{ Total } & 240 & 572 & 6.781 & 7.593 \\
\hline & $100 \%$ & $100 \%$ & $100 \%$ & $100 \%$ \\
\hline
\end{tabular}

Fonte: elaborado pelos autores com base em dados primários obtidos em formulário eletrônico

Tabela 3 - Relação entre o tempo a mais que está disposto a estar em auto isolamento e a percepção da qualidade da residência dos respondentes.

\begin{tabular}{|c|c|c|c|c|c|c|}
\hline \multirow{2}{*}{$\begin{array}{l}\text { Tempo a mais } \\
\text { disposto a estar em } \\
\text { auto isolamento }\end{array}$} & \multicolumn{6}{|c|}{ Qualidade da Residência } \\
\hline & Péssima & Ruim & Regular & Boa & ima & otal \\
\hline \multirow[t]{2}{*}{ Menos de 1 Mês } & 23 & 64 & 291 & 527 & 295 & 1.200 \\
\hline & $43,40 \%$ & $34,41 \%$ & $22,37 \%$ & $15,31 \%$ & $11,31 \%$ & $15,81 \%$ \\
\hline \multirow[t]{2}{*}{ De 1 a 2 Meses } & 9 & 34 & 299 & 747 & 442 & 1.531 \\
\hline & $16,98 \%$ & $18,28 \%$ & $22,98 \%$ & $21,70 \%$ & $16,95 \%$ & $20,17 \%$ \\
\hline \multirow[t]{2}{*}{ Mais de 2 Meses } & 2 & 8 & 44 & 108 & 86 & 248 \\
\hline & $3,77 \%$ & $4,30 \%$ & $3,38 \%$ & $3,14 \%$ & $3,30 \%$ & $3,27 \%$ \\
\hline \multirow[t]{2}{*}{ Tempo necessário } & 19 & 80 & 667 & 2.061 & 1.785 & 4.612 \\
\hline & $35,85 \%$ & $43,01 \%$ & $51,27 \%$ & $59,86 \%$ & $68,44 \%$ & $60,76 \%$ \\
\hline \multirow[t]{2}{*}{ Total } & 53 & 186 & 1.301 & 3.443 & 2.608 & 7.591 \\
\hline & $100 \%$ & $100 \%$ & $100 \%$ & $100 \%$ & $100 \%$ & $100 \%$ \\
\hline
\end{tabular}

Teste Qui-quadradado: $X^{2}=219,68 ; p<0.001$

Fonte: elaborado pelos autores com base em dados primários obtidos em formulário eletrônico.

Essa fato é de especial importância, como já demonstrado em outras situações que exigiram isolamento social ou quarentena, a situação de acesso inadequado a suprimentos básicos como comida, água, roupas ou acomodação foi uma fonte de frustração e continuava associado à DOl:http://dx.doi.org/10.14393/Hygeia0054397 Hygeia Edição Especial: Covid-19, Jun./2020 p.143 - 152, pág. 148 
ansiedade e à raiva alguns meses após a fim do período (BROOKS et al., 2020). Ainda mais quando considerada a realidade do estado de Pernambuco com mais de 875 mil pessoas vivendo em assentamentos subnormais (IBGE, 2020) e a detecção do material genético do SARS-COV-2 em esgotos não tratados e águas residuais (AHMED et al 2020) que apresentam potencial para permitir ou facilitar a transmissão de vírus de propagação aérea (GORMLEY et al ,2020).

Ainda que a classificação entre péssima e ótima seja bastante subjetiva, alguns elementos podem evidenciar a realidade dos aspectos de uma residência que facilitem a permanência em seu interior por um período mais longo. Dentre esses elementos podemos destacar a existência de uma área aberta ou área verde, como quintal, jardim ou varanda que alivie a sensação de encarceramento e propicie realizar atividades de recreação e lazer que ajudam a passar o tempo. Isso ficou evidenciado ao se relacionar a disposição em permanecer mais tempo em auto isolamento com a percepção se a existência de área aberta/verde na residência ajuda a permanecer na residência. Para $63,9 \%$ (3.616) dos respondentes ter esse tipo de estrutura tem contribuído significativamente para permanência de um maior tempo em auto isolamento (Tabela 4).

Esses dados levantam a questão acerca das condições das habitações no estado, como um fator de influência sobre a decisão por adotar o auto isolamento.

Tabela 4 - Relação entre o tempo a mais que está disposto a estar em auto isolamento e a percepção sobre os benefícios de ter área verde ou aberta em sua residência.

\begin{tabular}{lrrrr}
\hline & \multicolumn{5}{c}{ Áreas verde/aberta na casa ajuda } \\
\cline { 2 - 6 } $\begin{array}{c}\text { Tempo a mais disposto } \\
\text { a estar em auto } \\
\text { isolamento }\end{array}$ & \multicolumn{4}{c}{} \\
Não & \multicolumn{2}{c}{ Pouco } & Sim & Total \\
\hline Menos de 1 Mês & 146 & 350 & 394 & 890 \\
De 1 a 2 Meses & $32,74 \%$ & $21,96 \%$ & $10,90 \%$ & $15,74 \%$ \\
& 78 & 338 & 713 & 1.129 \\
Mais de 2 Meses & $17,49 \%$ & $21,20 \%$ & $19,72 \%$ & $19,96 \%$ \\
& 13 & 49 & 131 & 193 \\
Tempo necessário & $2,91 \%$ & $3,07 \%$ & $3,62 \%$ & $3,41 \%$ \\
& 209 & 857 & 2.378 & 3.444 \\
\hline Total & $46,86 \%$ & $53,76 \%$ & $65,76 \%$ & $60,89 \%$ \\
& 446 & 1.594 & 3.616 & 5.656 \\
\hline
\end{tabular}

Teste Qui-quadradado: $X^{2}=220,79 ; p<0.001$

Fonte: elaborado pelos autores com base em dados primários obtidos em formulário eletrônico.

\section{CONCLUSÕES}

O objetivo da pesquisa foi detectar os fatores que influenciam na decisão pelo auto isolamento, e desta forma tentar compreender os possíveis impactos oriundos desse processo. Neste sentido, observou-se que há uma crença majoritária da população amostrada na estratégia do isolamento social como medida de proteção à propagação do vírus, entretanto pesquisas posteriores já demonstram que esse apoio popular tende a diminuir com o tempo.

Fatores importantes, como as condições habitacionais e o número de pessoas na residência aparecem como fundamentais para entendermos melhor a propensão ou não ao isolamento social, pois são variáveis que influenciam bastante nas escolhas nesse contexto. Variáveis relacionadas a saúde, física e mental merecem estudos particulares, pois as correlações entre o auto isolamento e o estresse, a prática de atividades físicas e a qualidade do sono são significativas, e provavelmente continuarão tendo um impacto no período pós-pandemia. 
Os impactos ocasionados no convívio social têm uma relação direta com a saúde mental das pessoas, mas o impacto na renda tem uma repercussão direta na sobrevivência. Por isso, as medidas de apoio emergencial são fundamentais nesse momento de crise. As autoridades devem se preocupar em garantir o mínimo de condições para que as pessoas possam aderir ao isolamento e diminuir a curva de contágio. Além disso, as ações de comunicação devem superar o discurso do "fique em casa" para instigar as pessoas sobre as possibilidades do que fazer em casa no período do isolamento. Essas ações em conjunto são essenciais para mitigar os danos do isolamento durante e no período pós-pandêmico.

Por fim, ressaltamos que as pesquisas de opinião em contextos de crise são importantes para apresentar um retrato de como uma parte da população está vivenciando o período. Entretanto, a velocidade de expansão da Pandemia acarreta uma série de novas informações e ações, que podem modificar de forma rápida a percepção das pessoas sobre o problema em curso. Percepção esta, que precisa ser reforçada por informações claras e não contraditórias repassadas pelas autoridades públicas.

\section{REFERÊNCIAS}

AHMED, W; ANGEL, N; EDSON, J; BIBBY, K; BIVINS, A; O'BRIEN, J. W.; CHOI, P.I M.; KITAJIMA, M.i; SIMPSON, S. L.; LI, J.; TSCHARKE, B.; VERHAGEN, R; SMITH, W. J.M.; ZAUGG, J.; DIERENS, L.; HUGENHOLTZ, P; THOMAS, K. V.; MUELLER, J. F. First confirmed detection of SARS-CoV-2 in untreated wastewater in Australia: A proof of concept for the wastewater surveillance of COVID-19 in the community. Science of The Total Environment. p. 138764, 2020. Available at: https://doi.org/10.1016/j.scitotenv.2020.138764. https://doi.org/10.1016/j.scitotenv.2020.138764

ANDERSON, R. M. et al. How will country-based mitigation measures influence the course of the COVID-19 epidemic?. The Lancet, v. 395, n. 10228, p. 931-934, 2020. Acesso em 20 de abril de 2020. https://doi.org/10.1016/S0140-6736(20)30567-5

ATCHIMSON C, BOWMAN L, VRINTEN C, REDD R, PRISTERA P, EATON JW, WARD H. Perceptions and behavioural responses of the general public during the COVID-19 pandemic: A crosssectional survey of UK Adults. medRxiv 2020.04.01.20050039;

https://doi.org/10.1101/2020.04.01.20050039

BRASIL. Ministério da Saúde - Secretaria de Vigilância em Saúde. Boletim Epidemiológico Especial/COE-COVID-19. Disponível em: https://portalarquivos.saude.gov.br/images/pdf/2020/April/27/2020-04-27-18-05h-BEE14-Boletim-doCOE.pdf. Acesso em: 27 de abril de 2020.

BRASIL Ministério da Saúde (MS). Conselho Nacional de Saúde. Resolução n 510 , de 7 de abril de 2016. Dispõe sobre as normas aplicáveis a pesquisas em Ciências Humanas e Sociais. Diário Oficial da União 2016; 24 mai.

BRASIL. Instituto Brasileiro de Geografia e Estatística. Pesquisa Nacional por Amostra de Domicílios TIC 2018 / IBGE - Rio de Janeiro: IBGE, 2020. Disponível em: https://agenciadenoticias.ibge.gov.br/agencia-sala-de-imprensa/2013-agencia-de-

noticias/releases/27515-pnad-continua-tic-2018-internet-chega-a-79-1-dos-domicilios-do-pais. Acesso em: 29 de abril de 2020.

BRISCESE G, LACETERA N, MACIS S. M, TONIN M. Compliance with covid-19 social-distancing measures in italy: the role of expectations and duration. Nber Working Paper Series. Cambridge, MA: 2020. Available from: http://www.nber.org/papers/w26917 https://doi.org/10.3386/w26916

BROOKS, S. K. WEBSTER RK, SMITH LE, WOODLAND L, WESSELY S, GREENBERG N, RUBIN GJ. The psychological impact of quarantine and how to reduce it: rapid review of the evidence. The Lancet, v. 395, n. 10227, p. 912-920, 2020. Available from:https://doi.org/10.1016/S01406736(20)30460-8. 2020, April 15. https://doi.org/10.1016/S0140-6736(20)30460-8

CRODA J.H.R.; GARCIA L.P. Immediate Health Surveillance Response to COVID-19 Epidemic. Epidemiol. Serv. Saúde, Brasília. $\quad$ Available from $<$ http://www.scielo.br/scielo.php?script=sci_arttext\&pid=S2237-

96222020000100100\&lng=en\&nrm=iso>. access on 14 Apr. 2020. Epub Mar 23, 2020. https://doi.org/10.5123/S1679-49742020000100021 
DATAFOLHA. Opinião sobre a pandemia do Coronavírus: comportamento da população e medidas do governo. São 2020.2 Daulo, em: http://media.folha.uol.com.br/datafolha/2020/04/06/6c9855d692b869f13c5d83c421568342hb.pdf

DIÁRIO DE PERNAMBUCO. Pernambuco tem o segundo maior isolamento do país. Disponível em: https://www.diariodepernambuco.com.br/noticia/economia/2020/04/pernambuco-tem-o-segundomaior-isolamento.html. Acesso em 30 de abril de 2020.

ENDO, P. T; SILVA, I; LIMA, L; BARROS, M. H. F; DANTAS, M. R; ALVES I. R. R. Nota Técnica Monitorando as tendências de isolamento social em Pernambuco e na Região Metropolitana do Recife (RMR). Disponível em: https://jornalggn.com.br/a-grande-crise/fiqueemcasa-monitorando-astendencias-de-isolamento-social-em-pernambuco-e-na-regiao-metropolitana-do-recifermr/amp/? twitter impression=true. Acesso em: 29 de abril de 2020.

FETZER, T et al. Global Behaviors and Perceptions in the COVID-19 Pandemic. 2020. Disponível em: 10.31234/osf.io/3kfmh. Acesso em 23 de abril de 2020.

GARCIA LP, DUARTE E. Intervenções não farmacológicas para o enfrentamento à epidemia da COVID-19 no Brasil. Epidemiologia e Serviços de Saúde [online]. 2020. v. 29, n. 2. https://doi.org/10.5123/S1679-49742020000200009

GOOGLE. COVID-19 Community Mobility Report: Brazil April 17, 2020 Mobility changes. Los Angeles: Report, 2020. available at: https://www.google.com/covid19/mobility/

GORMLEY, M.; ASPRAY, T. J..; KELLY, D. A. COVID-19: mitigating transmission via wastewater plumbing systems. The Lancet Global Health, vol. 8, no. 5, p. e643, 2020. DOI 10.1016/S2214109X(20)30112-1. Available at: http://dx.doi.org/10.1016/S2214-109X(20)30112-1. https://doi.org/10.1016/S2214-109X(20)30112-1

INSTITUTO LOCOMOTIVA. Pesquisa sobre impacto da Covid-19. São Paulo, 2020. Disponível em: https://www.ilocomotiva.com.br/single-post/2020/04/14/ESTADO-DE-SPAULO-Novo-

coronav\%C3\%ADrus-reduz-renda-de-metade-dos-brasileiros-diz-pesquisa. Acesso em 25 de abril de 2020.

IPSOS. Opinião Pública sobre a Pandemia de Covid-19. São Paulo, 2020. Disponível em: https://www.ipsos.com/pt-br/56-dos-brasileiros-acreditam-que-isolamento-social-nao-impedirapropagacao-do-coronavirus. Acesso em 27 de abril de 2020.

LIMA, D.L.F, DIAS, A.A., RABELO, R.S, CRUZ, I.D, COSTA, S.C, NIGRI, F.M.N, NERI, J.R. COVID19 no Estado do Ceará: Comportamentos e crenças na chegada da pandemia. Cien Saude Colet [periódico na internet] (2020/Abr). Disponível em: http://www.cienciaesaudecoletiva.com.br/artigos/covid19-no-estado-do-ceara-comportamentos-ecrencas-na-chegada-da-pandemia/17540?id=17540\&id=17540.

https://doi.org/10.1590/SciELOPreprints.14

MORALES AJ, GALLEGO V, ESCALERA-ANTEZANA JP, MÉNDEZ CA, ZAMBRANO LI, FRANCOPAREDES C, SUÁREZ JA, RODRIGUEZ-ENCISO HD, BALBIN-RAMON GJ, SAVIO-LARRIERA E, RISQUEZ A, CIMERMAN S. COVID-19 in Latin America: The implications of the first confirmed case in Brazil. Travel Medicine and Infectious Disease, 2020. https://doi.org/10.1016/j.tmaid.2020.101613

OLIVEIRA A, VILELA S, WARKENTIN S, ARAÚJO J, RAMOS E, LOPES. COVID-19: Comportamentos alimentares e outros estilos de vida saudáveis em tempo de isolamento social. In: Da emergência de um novo vírus humano à disseminação global de uma nova doença - Doença por Coronavírus 2019 (e-book). Instituto de Saúde Pública da Universidade do Porto (ISPUP), Portugal, 2020. Disponível em: https://ispup.up.pt/news/internal-news/boletim-de-publicacoes-covid19/916.html/?lang=pt. Acesso em: 28 de abril de 2020.

ORNELL F, SCHUCH J. B, SORDI A. O, KESSLER F. H. P. Pandemia de medo e Covid-19: Impacto na saúde mental e possíveis estratégias. Revista debates in psychiatry. 2020. In press. Disponível em: http://www.ufrgs.br/ufrgs/noticias/arquivos/pandemia-de-medo-e-covid-19-impacto-na-saudemental-e-possiveis-estrategias 
PANCANI L, MARINUCCI M, AURELI N, RIVA P. Forced social isolation and mental health: A study on 1006 Italians under COVID-19 quarantine. Doi: 10.31234/osf.io/uacfj. https://doi.org/10.31234/osf.io/uacfj

PERNAMBUCO. Secretaria de Saúde do Estado. Nota Técnica SES/PE No03/2020 | Novo Coronavírus (COVID-19). Disponível em: https://12ad4c92-89c7-4218-9e110ee136fa4b92.filesusr.com/ugd/3293a8_5ffd810e04734cbc82de92b7f375263a.pdf. Acesso em: 26 de abril de 2020.

PERNAMBUCO. Legislação do Estado de Pernambuco. Decreto N. 48.809 de 14 de março de 2020. [Online]. Disponível em: https://legis.alepe.pe.gov.br/texto.aspx?id=49417\&tipo= Acesso em: 26 de abril de 2020.

PERNAMBUCO. Secretaria Estadual de Saúde. Informe Epidemiológico N. 56/2020. Disponível em: https://12ad4c92-89c7-4218-9e11-

0ee136fa4b92.filesusr.com/ugd/3293a8_b494e8ac48d841abbd95eb5d717f9425.pdf. Acesso em: 27 de abril de 2020.

PORTAL COVID-19 BRASIL. Análise Subnotificação. Disponível em https://ciis.fmrp.usp.br/COVID19/analise-subnotificacao/. Acessado em: 13/04/2020.

QIU J, SHEN B, ZHAO M. WANG Z, XIE B, XU Y. A nationwide survey of psychological distress among Chinese people in the COVID-19 epidemic: implications and policy recommendations. General Psychiatry 2020;33:e100213. https://doi.org/10.1136/gpsych-2020-100213

XIAO H., ZHANG Y., KONG D., LI S., YANG N. Social capital and sleep quality in individuals who selfisolated for 14 days during the coronavirus disease 2019 (COVID-19) outbreak in January 2020 in China. Med. Sci. Monit., 26 (2020). p. e923921-1, 2020. https://doi.org/10.12659/MSM.923921

WORLD HEALTH ORGANIZATION. New Coronavirus - China. Disponível em: https://www.who.int/csr/don/12-january-2020-novel-coronavirus-china/en/. Acesso em 23 de abril de 2020. 